\title{
Differential modulation of corticospinal excitability during observation, mental imagery and imitation of hand actions
}

\author{
Shannon Clark ${ }^{a}$, François Tremblay ${ }^{b, *}$, Diane Ste-Marie ${ }^{a}$ \\ a School of Human Kinetics, Faculty of Health Sciences, University of Ottawa, Ottawa, Ont., Canada K1H 8 M5 \\ ${ }^{\mathrm{b}}$ School of Rehabilitation Sciences, Faculty of Health Sciences, University of Ottawa, 451 Smyth Road, Ottawa, Ont., Canada K1H $8 M 5$
}

Received 28 November 2002; received in revised form 14 January 2003; accepted 27 May 2003

\begin{abstract}
In this study, we attempted to better delineate the changes in corticospinal excitability that accompany perceptual to motor transformations when people are asked to observe, image or imitate actions. Motor evoked potentials (MEP) from transcranial magnetic stimulation were recorded in the first dorsal interosseous (FDI) muscle of the dominant hand (15 right, 4 left) in five different conditions: (1) passive observation; (2) observation to imitate; (3) imagery; (4) imitation; and (5) counting backwards mentally. MEPs were also recorded at rest at the beginning and at the end of the session to establish baseline (BL) values. For the observation conditions, participants $(n=19,18-38$ years) watched video sequences $(5 \mathrm{~s}$ ) of hand actions performed by a model with the right arm (passive observation: scissors; observation to imitate: OK sign). Active imitation produced the greatest MEP facilitation compared to baseline, followed by the two observation conditions and the imagery conditions, which all produced similar levels of facilitation (post hoc comparisons). Mental counting produced some facilitation, but this effect was inconsistent. Baseline MEPs remained stable at the end of the session. A further comparison between right-handers $(n=15)$ and left-handers $(n=4)$ revealed no difference in the pattern of modulation across conditions. The similarity found between observation and imagery of hand actions in terms of corticospinal facilitation is interpreted in the light of the motor-simulation theory of Jeannerod [Neuroimage 14 (2001)], which proposes that perceiving actions involves neural simulation of the same action by the observer, thereby explaining the parallel between actions observed and actions imaged at the representational level.
\end{abstract}

(C) 2003 Elsevier Ltd. All rights reserved.

Keywords: Perceptual-motor interaction; Motor imagery; Action observation; Motor cortex; Transcranial magnetic stimulation

\section{Introduction}

Observing a model and mental imagery are commonly used methods to promote skill acquisition. Indeed, through observing demonstrations of skilled performances, one can derive cues that can be used subsequently to reproduce the intended action. Similarly, through mental rehearsal of previously learned actions, or parts of actions, one can prepare the sensorimotor apparatus for optimal performance in a subsequent execution. Such training strategies generally reflect traditional assumptions about the existence of links between the perceptual and motor systems. For instance, Meltzoff and Moore's (1977) active intermodal matching theory postulates that humans possess an innate capacity to imitate actions through a dedicated mechanism that transforms visual output of the model into motor output via supramodal representations. In another competing

\footnotetext{
* Corresponding author. Tel.: +1-613-562-5800; fax: +1-613-562-5428.

E-mail address: ftrembla@uottawa.ca (F. Tremblay).
}

theory, Heyes (2001) emphasizes the existence of a set of bi-directional excitatory links between sensory and motor representations that are formed largely from correlated experiences of observing and executing actions. Likewise, theories of motor imagery have been embedded within the same conceptual framework with regard to the transformation of perceived or learned actions into motor images and motor performance (Annett, 1996; Jeannerod \& Frak, 1999).

From a neurobiological perspective, the perceptual-motor translation problem has been highlighted in recent years through brain imaging techniques (PET and fMRI) and electrophysiological methods. From converging evidence of neuroimaging studies in humans and neuronal recordings in non-humans primates, a picture has emerged indicating that the neural circuitry involved in action execution overlaps extensively with that activated when actions are imaged or observed (see Decety and Grèzes (1999); Grezes and Decety (2001) for recent reviews). The shared neural network includes the premotor cortex, supplementary motor area (SMA), the inferior parietal lobule, cingulated 
gyrus, and the cerebellum (Decety \& Grèzes, 1999). Activation of the primary motor cortex has also been reported during motor imagery tasks (Pfurtscheller \& Neuper, 1997; Porro, Francescato, \& Cettolo, 1996) and during action observation (Hari et al., 1998). Further evidence for the involvement of the primary motor cortex is derived from studies relying on the technique of transcranial magnetic stimulation (TMS). With this technique, dynamic modulations of corticospinal excitability have been described in hand muscles when participants observed (Fadiga, Fogassi, Pavesi, \& Rizzolatti, 1995; Strafella \& Paus, 2000) or imaged (Abbruzzese, Trompetto, \& Schieppati, 1996; Hashimoto \& Rothwell, 1999; Kasai, Kawai, Kawanishi, \& Yahagi, 1997) hand actions. Such results clearly established that the neural structures engaged in motor execution are also active when actions are observed or imaged.

TMS explorations have further indicated that changes of corticospinal excitability during action observation and motor imagery are largely specific to the muscles involved in the observed (Maeda, Kleiner-Fisman, \& Pascual-Leone, 2002; Strafella \& Paus, 2000) or imaged (Fadiga et al., 1999; Rossini, Rossi, Pasqualetti, \& Tecchio, 1999) action. To our knowledge, however, no TMS studies to date have actually compared the changes in corticospinal excitability that occur when participants are required to observe actions versus observe in order to subsequently image or imitate the previously seen actions. There is evidence both from behavioural and neuroimaging studies that participant's intentions do influence attentional processes (Mataric \& Pomplun, 1998) and patterns of cortical activation (Decety, Grezes, \& Costes, 1997; Grezes, Costes, \& Decety, 1999), during action observation. The importance of cognitive factors in the transformation of perceptions into actions has been further emphasized recently in the theoretical framework developed by Jeannerod (2001) on motor simulation. According to this theory, attending to another person's actions involves the neural simulation of the same perceived action, thus explaining the equivalence between action imaged and action observed in terms of neural processing.

In the present study, our goal was to better delineate the changes in corticospinal excitability in hand muscles that accompany perceptual to motor transformations when participants are asked to observe, image and imitate specific hand actions.

\section{Method}

\subsection{Participants}

The participants consisted of 19 healthy individuals, 6 males and 13 females, between the ages of 18 and 38 years (mean, $24.8 \pm 8.9$ years). Four participants were left-handed and 15 were right-handed. Prior to the experimental session, all participants completed a medical questionnaire to ensure that they did not have any contraindications for transcranial magnetic stimulation. The local Institutional Ethics Committee approved the study's procedures and each participant provided informed consent before participation.

\subsection{TMS and recording of motor evoked potentials}

For magnetic stimulation, the participants were seated comfortably in a recording chair, with their arms and hands resting on armrests and their feet supported by a stool. A U-shaped cushion was placed around the neck to ensure participants' comfort and also to limit head movements during the experiment. Magnetic stimulation was delivered with a MagStim 200 (The MagStim Co., Dyfed, UK) connected to a figure-of-eight coil $(70 \mathrm{~mm}$ loops). To record motor evoked potentials (MEP), small auto-adhesive surface electrodes $\left(1 \mathrm{~cm}^{2}\right)$ were placed in a belly-tendon bipolar montage over the first dorsal interosseous (FDI) muscle of the dominant hand (i.e. hand for writings). The electromyographic (EMG) signals were amplified $(100 \mu \mathrm{V} / \mathrm{div})$ and filtered (bandwidth, $10 \mathrm{~Hz}$ to $5 \mathrm{kHz}$ ) with a polygraph amplifier (RMP-6004, Nihon-Kohden Corp.). For each trial, $100 \mathrm{~ms}$ epochs were recorded with a $5 \mathrm{~ms}$ delay preceding stimulus onset. EMG signals were digitized at a $2 \mathrm{kHz}$ sampling rate using custom software on a PC running under Microsoft ${ }^{\circledR}$ Windows ${ }^{\circledR} 98$ equipped with a digital/analog acquisition card (BNC-2090, National Instrument Corp.).

\subsection{Determination of the relaxed motor threshold and baseline MEPs}

The first step in the TMS session consisted of localizing the optimal site on the scalp to evoke responses in the contralateral FDI muscle. To this end, the coil (intersection site) was placed over the approximate location of the hand motor area on the left $(n=15)$ or right $(n=4)$ hemi-scalp (i.e. $\sim 4-5 \mathrm{~cm}$ laterally from the vertex on the inter-aural line (Mills \& Nithi, 1997; Weber \& Eisen, 2002). With the handle oriented $\sim 45^{\circ}$ in the mid-sagittal plane and the stimulator set at $60 \%$ of its maximal output, the target area was systematically explored by displacing the coil in small steps until large responses could be evoked in the contralateral FDI. Once the optimal spot was localized, the site was marked with a red dot to ensure consistent coil positioning. In all sessions, the same experimenter (F.T.) was responsible for holding the coil in place for the duration of the experiment, which lasted $\sim 20-25 \mathrm{~min}$. When the coil had to be moved in some rare occasions, for instance to accommodate participants (e.g. for coughing), the experimenter used the red dot and other marks on the scalp and/or the ear to reposition the coil over the same site.

The second step consisted of determining the relaxed motor threshold using the method outlined by Mills and Nithi (1997). Starting from supra-threshold intensity, the stimulator's output was gradually decreased in $1 \%$ steps until no MEPs could be evoked for 10/10 consecutive stim- 
(A) HAND ACTION: PASSIVE OBSERVATION
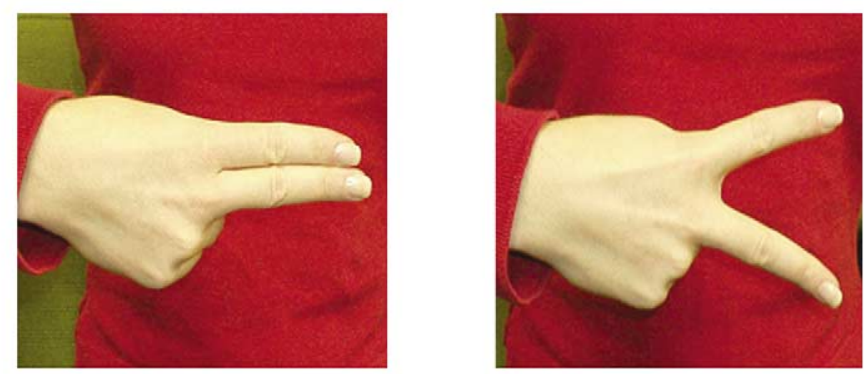

(B) HAND ACTION: OBSERVATION FOR IMITATION
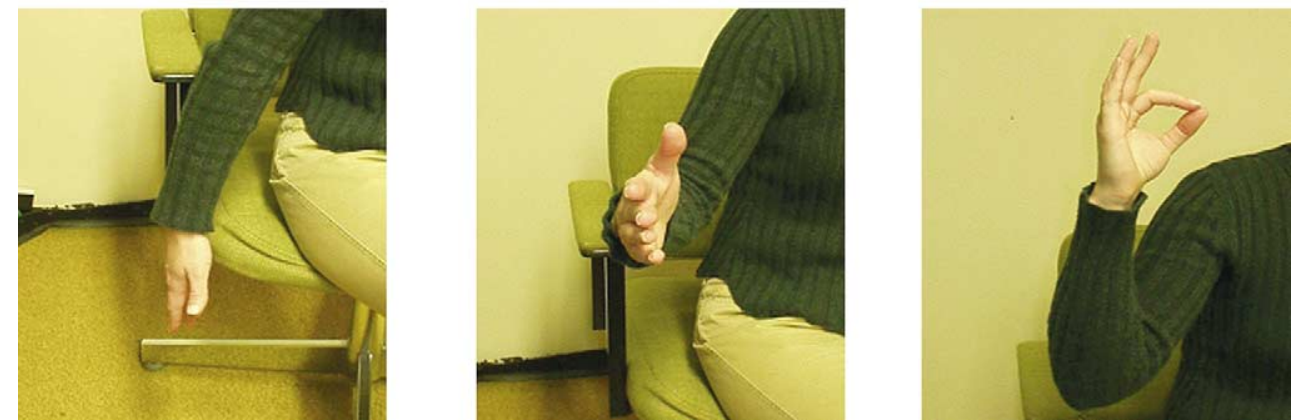

Fig. 1. Photography snapshots of the videotaped pantomime actions that were presented to participants in the two observation conditions. Note that all participants could spontaneously identify the meaning of each action (A) scissors-like movement; (B) OK sign.

uli. This TMS intensity corresponded to the lower threshold value. From this point, the intensity was gradually increased until MEPs of at least $50 \mu \mathrm{V}$ peak-to-peak amplitude could be evoked in 10/10 consecutive stimuli. The latter intensity determined the upper threshold value. The relaxed motor threshold was defined for each participant as the median intensity between the upper and lower threshold values (Mills \& Nithi, 1997).

The final step was to establish baseline (BL) MEP values. For baseline measurements, 10 MEPs were recorded in the FDI in response to supra-threshold stimuli (threshold $+10 \%$ of stimulator output) with participants being instructed to fully relax their muscles.

\subsection{Observation, imagery and imitation of a hand action}

The second part of the TMS session consisted of measuring MEPs consecutively in six different conditions:

1. observation of a hand action without prior instructions (passive observation);

2. observation of a hand action with prior instructions as to a subsequent imitation or imagery of the observed action (observation to imitate);

3. imagery of the hand action previously observed (imagery);

4. actual reproduction of the hand action previously observed (imitation);

5. counting backwards mentally (counting);

6. post-baseline.
In the first two observation conditions, participants watched videotaped performances of hand actions executed by a model (right arm) on a $27 \mathrm{in}$. TV set placed $\sim 1.5 \mathrm{~m}$ directly in front of the participant. For the first condition, participants were simply asked to observe a simple hand action (scissors action, $5 \mathrm{~s}$ duration, Fig. 1A). For the observation with the intent to imitate, the participants viewed a different hand action (OK sign, $5 \mathrm{~s}$ duration, Fig. 1B), however, this time they were informed that they would be required to imitate and image the same action shortly after. For the two observation conditions, each video sequence of the action was presented 10 times with a $10 \mathrm{~s}$ interval between each presentation. In each visioning, TMS was triggered manually (intensity $=$ threshold $+10 \%$ ) so as to coincide with the onset of a specific movement sequence in the task being observed (i.e. finger abduction@ $2.5 \mathrm{~s}$ in the scissors action and thumb-index pinching action@3s in the OK sign). For the imagery condition, the participants were asked to image the hand action they had viewed in the preceding trials (i.e. OK sign). The experimenter provided the cue as to when to start imaging and the participants indicated when they had completed the imaged action. The experimenter used a stopwatch to determine when to deliver TMS in the imagery trials so the delivery occurred at approximately the same time as in the observation to imitate condition (i.e. pinching action @ $3 \mathrm{~s}$ ). For the imitation condition, the participants were asked to actually reproduce the hand action they had viewed and just imaged and TMS delivery was again appropriately timed to the onset of the 
same specific movement sequence (i.e. pinching action). For both the imagery and imitation conditions, 10 trials were performed. For the counting condition, the participants were asked to count backwards silently from 100 and TMS was delivered every $10 \mathrm{~s}$ for a total of 10 trials. Finally, to ensure that the stimulation conditions had remained stable throughout the experiment, MEPs at rest were re-assessed at the end of the session to determine post-baseline values.

In all conditions, and especially in those in which EMG silence was required (observation, imagery and counting), the FDI muscle activity was constantly monitored on an oscilloscope at high gain $(50 \mu \mathrm{V} / \mathrm{div})$ to ensure that full relaxation was maintained. Whenever unwanted EMG activity was detected, the trial was aborted and participants were reminded that relaxation was required. In the majority of participants tested, EMG silence was easily obtained. "Catch" trials were also introduced on some trials. This was accomplished by switching-off the stimulator unexpectedly while the participant observed or imaged the requested hand action in order to assess for the presence of unwanted EMG bursts (see Section 2).

\subsection{Data analysis and statistics}

The peak-to-peak amplitude and latency of MEPs recorded in each condition for each participant were measured off-line and averaged to derive individual mean

(A)
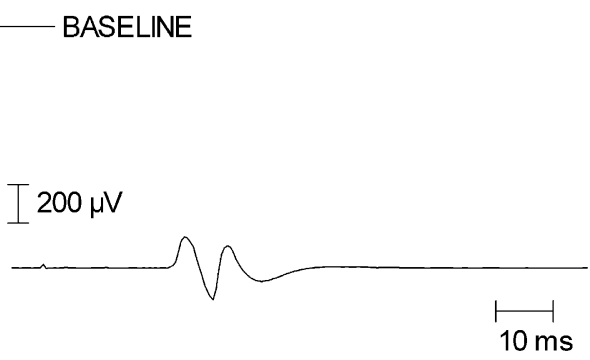

(C)
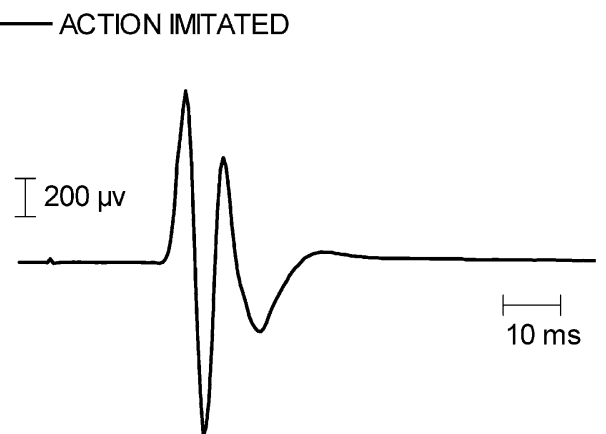

values. For the amplitude data, each individual mean value was transformed into natural logarithms in order to normalize the distribution and allow inter-individual comparisons (Nielsen, 1996). The individual mean log-amplitudes and latencies were then entered into a repeated measures one-way analysis of variance (ANOVA), with seven within-participants variables: (1) baseline; (2) passive observation; (3) observation to imitate; (4) imagery; (5) imitation; (6) counting; and (7) post-baseline. The Dunnett's post-test was used to detect significant differences between amplitude and latency measurements at baseline and those obtained for each of the six remaining conditions. The Bonferroni's multiple comparisons test was also used to compare conditions that were significantly different from baseline. All tests were performed using GraphPad Prism version 3.00 for Windows ${ }^{\circledR}$ (GraphPad Software, San Diego, CA, USA). The significance level was set at $P<0.05$ for all tests.

\section{Results}

\subsection{MEPs facilitation with observation, imagery and imitation}

The relaxed threshold derived from the upper and lower limits averaged $54 \%$ (S.D. $\pm 7 \%$ ) in our participants, with three-quarters of them having threshold values of $<60 \%$.
(B)
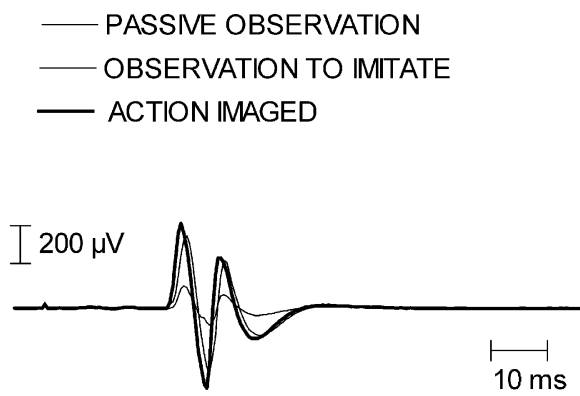

(D)

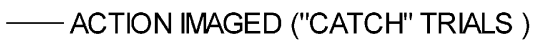

Fig. 2. Example of the pattern of modulation seen in the amplitude of motor evoked potentials (MEP) across the various testing conditions. "A" represents MEPs recorded at rest as baseline. In "B", MEPs recorded in three different conditions are superimposed. Note the amplitude facilitation relative to baseline in the observation to imitate and imagery conditions. "C" shows the large amplitude facilitation associated with active imitation. "D" represents catch "imagery trials" in which the participant was unaware that the simulator had been unarmed. Note the absence of any activity in the resting the FDI muscle. Each trace represents an average of 10 trials, with the exception of traces in "D" $(n=3)$. 
One individual (\#10, male 38 years) had an unusually high threshold (71\%) for the FDI. Right-handers and left-handers had similar averaged thresholds ( $54 \pm 7$ and $54 \pm 4 \%$, respectively). Comparison of MEP amplitude across the various testing conditions revealed a pattern of significant differential modulation $\left(F_{6,18}=20.4, P<0.001\right)$. An example of this pattern is shown in Fig. 2. It can be seen that passive observation did not have a large effect on MEP amplitude in this individual, whereas observation to imitate, action imagery and imitation (Fig. 2B and C) were all associated with MEP facilitation, albeit at different levels. Notice the lack of any significant EMG activity in the FDI for "catch" imagery trials (i.e. when the stimulator had been unexpectedly turned-off).

Fig. 3 shows the averaged MEP log-amplitudes (A) and latencies (B) computed for all participants across all condi-
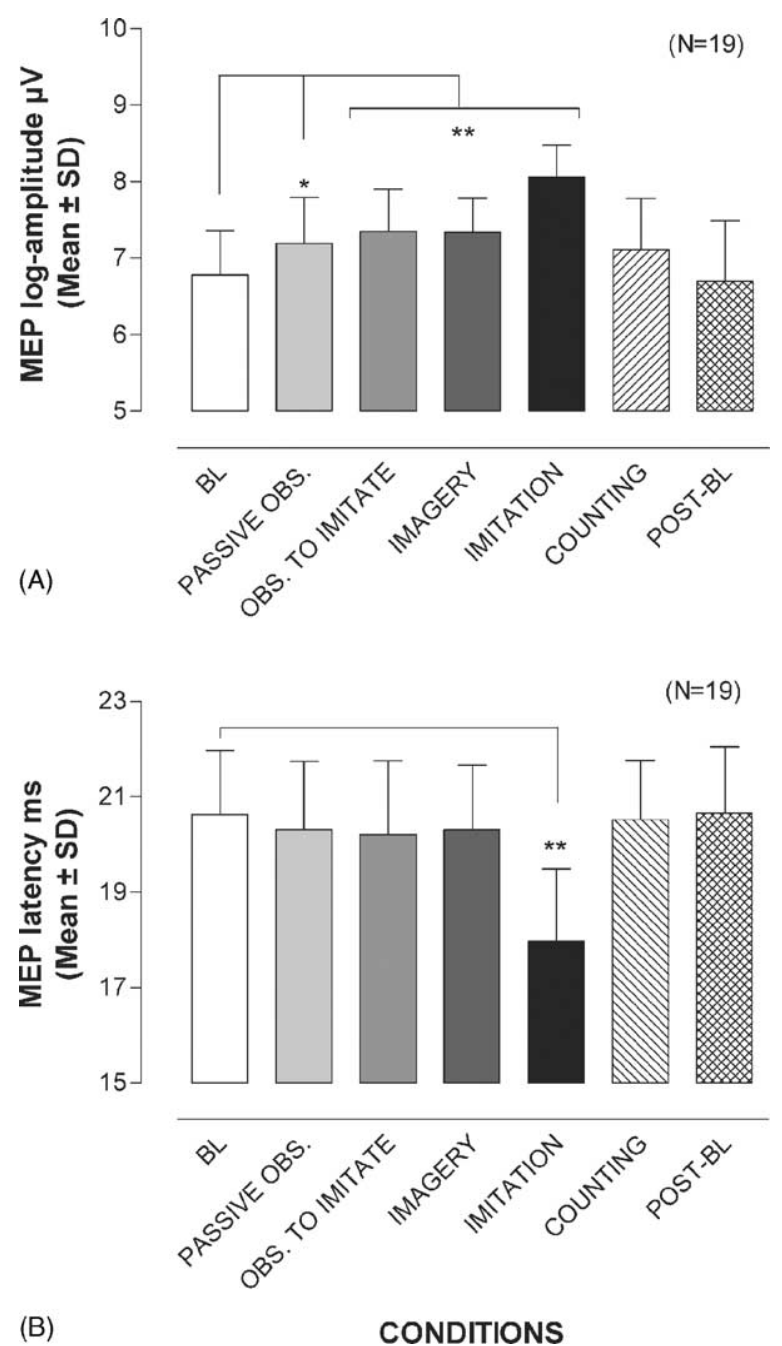

Fig. 3. Comparisons of the mean changes in MEP log-amplitudes (A) and MEP latencies (B) computed from all participants in the various testing conditions. The asterisks indicate levels of statistical significance derived from the Dunnett's post-test in comparing each condition against baseline (BL); (*) $P<0.05$, (**) $P<0.01$. The bar for post-BL corresponds to the mean values of baseline MEPs recorded at rest at the end of the session. tions. It can be seen that four conditions (i.e. passive observation, observation to imitate, imagery and imitation) were associated with significant MEP facilitation with respect to baseline (Dunnett's post-test, $P<0,05$ ). Of these, active imitation produced the greatest level of facilitation in the FDI muscle (Bonferroni's multiple comparisons test, $P<$ 0.001). The observation to imitate and imagery conditions produced slightly more amplitude facilitation than passive observation, but the differences between these conditions were not significant (Bonferroni's multiple comparisons test, $P>0.05$ ). Consistent with the amplitude facilitation observed in the four conditions, the corresponding MEP latencies measured were also decreased when compared to baseline. The difference was statistically significant, however, only in the active imitation condition (Fig. 3B). MEPs measured in the two remaining conditions (i.e. counting and post-baseline) did not differ significantly from those measured at baseline, although approximately half of the participants (9/19) showed evidence of facilitation in the mental counting task.

\subsection{Effect of hand dominance}

Since a significant proportion of our participants were left-handed (4/19), and given that lateralization could be an issue in the context of action observation and imitative behaviours (Aziz-Zadeh, Maeda, Zaidel, Mazziotta, \& Iacoboni, 2002; Fadiga et al., 1999; Iacoboni et al., 2001), we proceeded to re-examine the changes in corticospinal excitability just described with respect to hand dominance. Fig. 4 illustrates the average MEP amplitude (A) and latencies (B) measured at baseline and under the four "facilitatory" conditions with participants now sorted by handedness. As evident in Fig. 4, the pattern of modulation seen in the small group of left-handed participants did not differ from that seen in the majority of right-handed participants. The fact that hand dominance had no effect on the observed changes in corticospinal excitability was further confirmed when MEP amplitude data in each condition were submitted to ANOVA with handedness and gender as fixed factors and age as a co-variate. None of these factors came out as significant $\left(F_{1,14}<3.5, P>0.05\right)$.

\section{Discussion}

In the present study, we sought to better delineate the changes in corticospinal excitability that take place when individuals observe, image or imitate simple hand actions. Our results revealed the presence of consistent facilitatory effects only in conditions wherein participants were either mentally or physically engaged in motor processes, either through observation, explicit motor imagery or active imitation of hand actions. The counting condition, in which participants were engaged in a non-motor cognitive task, produced no consistent facilitation in our participants; ruling 

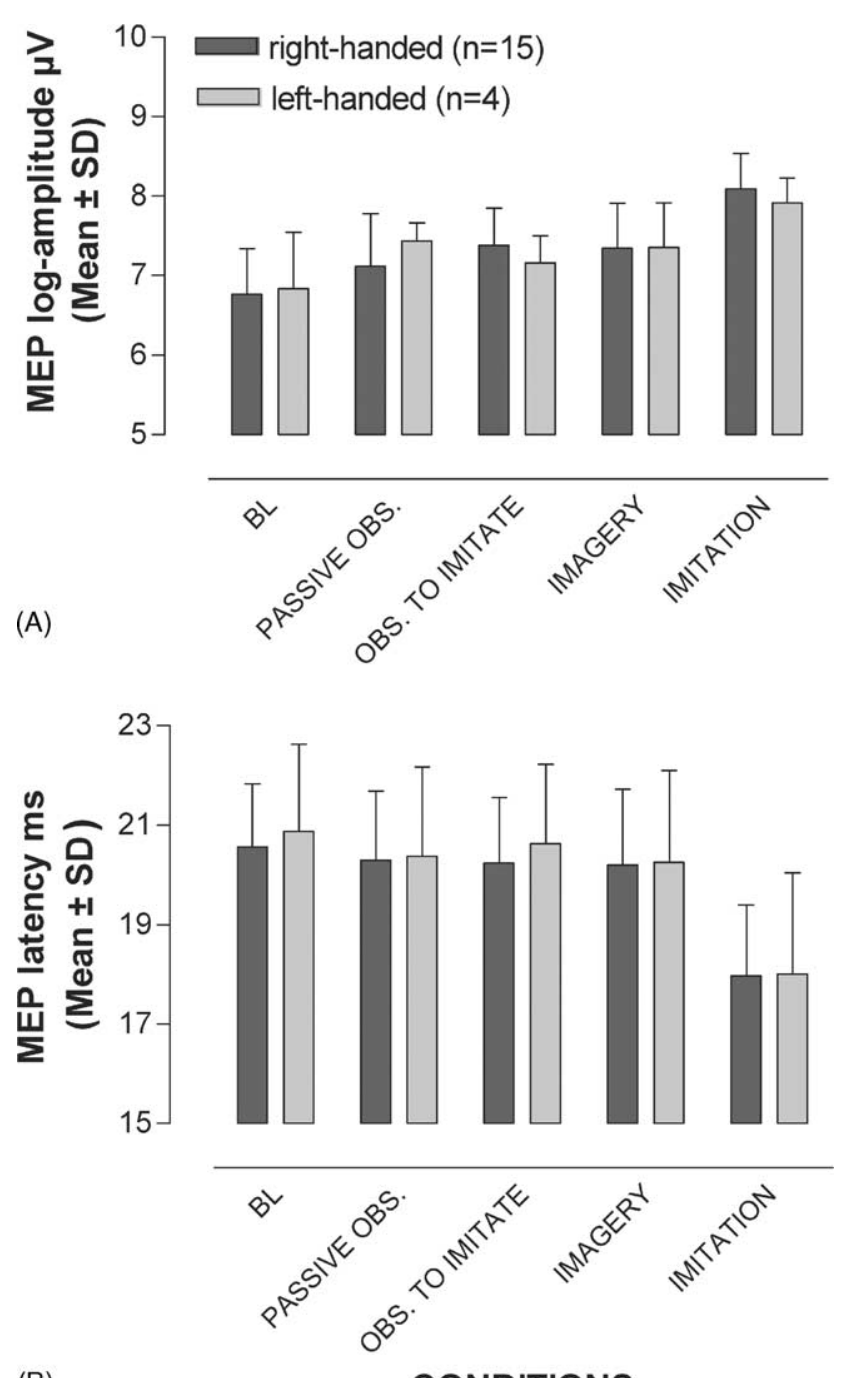

(B)

\section{CONDITIONS}

Fig. 4. Comparisons of the mean changes in MEP log-amplitudes (A) and MEP latencies (B) between right-handed and left-handed participants. Note that only five of the seven testing conditions are shown here. Abbreviations as in Fig. 3.

out the possibility that the reported effects could only be attributed to changes at the attentional level. Nevertheless, the fact that about half of the participants showed larger MEPs in the counting condition suggests that part of the facilitation seen during action observation and imagery tasks could have been related to attentional processing since activation of cortical motor areas has been reported in mental counting tasks involving sustained attention (Ortuno, Ojeda, \& Arbizu, 2002). On the other hand, the fact that baseline MEPs remained stable at the beginning and at the end of the session indicates that corticospinal excitability at rest was not altered in spite of the repeated stimulations and also attests for the reliability of the recording technique. Thus, in spite of some possible influences from attentional processes, it seems that most of the changes reported here reflected specific facilitatory influences exerted onto the corticospinal system in the context of perceptual to motor transformations.
Not surprisingly, the greatest facilitation was observed during actual imitations of the previously seen action. Voluntary contraction is known to produce the most potent facilitation of magnetically induced cortico-motor responses by increasing the number and size of descending volleys and, at the same time, lowering the firing threshold of spinal motoneurones via incoming peripheral afferents (Di Lazzaro, Restuccia, \& Oliviero, 1998; Mills \& Kimiskidis, 1996; Ugawa, Terao, Hanajima, Sakai, \& Kanazawa, 1995). The increased MEP amplitudes and the reduced latency exhibited by our participants during active imitation are, therefore, entirely consistent with a rise in excitability at the cortical and spinal level. The other conditions that produced significant facilitation of MEPs involved no overt muscle activity on the part of the participants, rather, these conditions simply involved observing or imaging specific hand actions while being at rest. As pointed out by Rossini et al. (1999), such observations suggest that corticospinal excitability to hand muscles can be "internally" modulated when people are mentally engaged in preparing or planning motor actions.

In accordance with earlier TMS studies (Fadiga et al., 1995; Strafella \& Paus, 2000), we found that corticospinal excitability increased when participants observed the performance of hand gestures. While the two actions were kinematically different, both involved activation of the FDI muscle in producing a familiar finger gesture, and thus accounted for the reported selectivity of the facilitation for muscles involved in the observed task (Fadiga et al., 1995; Strafella \& Paus, 2000). Interestingly, both actions also involved finger movements (i.e. finger aperture and closure) that have been associated recently with phase-specific modulation of corticospinal excitability during observed hands movements in humans (Gangitano, Mottaghy, \& Pascual-Leone, 2001; Maeda, Kleiner-Fisman, \& Pascual-Leone, 2002). In this respect, our results are entirely consistent with the notion that one's own corticospinal system is activated when observing others performing actions (Fadiga et al., 1995; Maeda et al., 2002).

The existence of a system in humans that allows matching of observed actions with their execution implies a certain degree of lateralization so that greater activation is expected in each hemisphere when observing actions performed by the contralateral extremity (i.e. mirror-image movements Fadiga et al., 1995; Iacoboni et al., 1999). Although we did not specifically address this question in our study, the presence of a significant proportion of left-handers (and of right hemisphere stimulations) allowed a comparison to be made. In this regard, we found no evidence of greater MEP facilitation in right-handed subjects watching hand movements performed in a mirror-image perspective. In a recent TMS study Aziz-Zadeh, Maeda, Zaidel, Mazziotta, and Iacoboni (2002) reported a stronger sensitivity of the right hemisphere for laterality effects, with significantly greater facilitation to presentation of left versus right hand stimuli. In our study, MEP amplitude facilitation was highly comparable between right-handers and left-handers when 
viewing video sequences of right hand actions. Although our observations seem at odds with the reported sensitivity of the right hemisphere, methodological differences in the format of action presentation could explain some of the discrepancy (e.g. hand orientations). Besides, the number of left-handers was too small in this study to draw any firm conclusions on the issue of laterality.

While there was some indication of larger amplitude facilitation in the observation to imitate versus passive observation condition (as judged by the higher significance level when compared to baseline), the MEP amplitudes in the two observation conditions were not significantly different. Thus, it seems that corticospinal excitability was only marginally affected when participants had received prior instructions as to a subsequent imitation of the action. This result is somewhat surprising considering the evidence that an individual's intention during action observation influences cognitive strategies and cerebral activation. Mataric and Pomplun (1998), for example, reported higher pupil dilation, reflecting higher attention to the movement, when individuals observed hand actions with the intent to imitate versus no imitations. In neuroimaging studies, additional activation was detected in motor areas involved in motor preparation and programming (e.g. SMA, cingulated gyrus) when subjects were requested to observe actions with the aim to imitate (Decety et al., 1997; Grezes et al., 1999). One possibility for the absence of consistent effects related to participants' intention in the present study might reside in the selection of the model action itself. Indeed, the concept of imitation has undergone many revisions over the recent years (review in Heyes, 2001). For instance, it has been proposed that novelty could be a necessary condition for genuine imitation to occurs, otherwise, observing others' action can simply act as a priming stimulus for retrieving a similar action rather than for copying of the model (Byrne \& Russon, 1998). As emphasized by Byrne and Russon (1998), observational priming still relies on perceptual to motor transformation as stored internal representations corresponding to the observed action need to be activated. Thus, it is possible that observation of the OK sign with the aim to imitate did not produce much facilitation beyond that associated with passive observation simply because its visioning only acts as a trigger to reproduce a movement already present in the individual's own repertoire. The novelty of the movement to be imitated is certainly an issue for future studies on corticospinal facilitation during action observation.

Also consistent with previous TMS studies (Fadiga et al., 1999; Rossi, Pasqualetti, Tecchio, Pauri, \& Rossini, 1998; Rossini, Rossi, Pasqualetti, \& Tecchio, 1999), MEPs in the FDI muscle were facilitated when participants performed mental simulation of the hand action. The level of MEP facilitation with imagery was comparable to that seen during the two observation conditions and all were smaller than active imitation. In neuroimaging studies, the level of motor cortex activation in imagery tasks has been reported to be typically lower than that seen during actual movement execution (Mellet, Petit, Mazoyer, Denis, \& Tzourio, 1998). This observation can account for the lower MEP amplitude facilitation found in the imagery and observation conditions. Of course, changes in excitability at the spinal level during imagery (Bonnet, Decety, Jeannerod, \& Requin, 1997) and action observation (Baldissera, Cavallari, Craighero, \& Fadiga, 2001) might have also influenced MEP amplitude under these conditions.

It is of particular interest that the two processes, action observed and action imaged, led to comparable levels of corticospinal facilitation in the FDI. Such a finding is consistent with the motor-simulation theory according to which perceiving actions involves internal simulation of the movement to be produced (Jeannerod, 2001). Thus, when our participants observed the action, presumably, they simulated the action internally, much like what they did when explicitly asked to image doing the same action. The parallel found between imagery and observation in terms of corticospinal facilitation emphasizes the similitude of the two processes when used to promote skills learning. In view of the evidence at the behavioural level, that high ability imagers are actually better than low ability imagers in learning new motor skills (see Hall, Buckolz, and Fishburne (1992) for a review), it would be interesting to determine whether an individual's ability to image (e.g. as a result of training) could influence the level of corticospinal facilitation seen while action are observed or imaged. This issue is currently under investigation in our lab.

In conclusion, the present study provides insightful data that further delineates the differential effects of action observation, imagery, and action imitation on corticospinal excitability to hand muscles.

\section{References}

Abbruzzese, G., Trompetto, C., \& Schieppati, M. (1996). The excitability of the human motor cortex increases during execution and mental imagination of sequential but not repetitive finger movements. Experimental Brain Research, 111, 465-472.

Annett, J. (1996). On knowing how to do things: A theory of motor imagery. Cognitive Brain Research, 3, 65-69.

Aziz-Zadeh, L., Maeda, F., Zaidel, E., Mazziotta, J., \& Iacoboni, M. (2002). Lateralization in motor facilitation during action observation: A TMS study. Experimental Brain Research, 144, 127-131.

Baldissera, F., Cavallari, P., Craighero, L., \& Fadiga, L. (2001). Modulation of spinal excitability during observation of hand actions in humans. European Journal of Neuroscience, 13, 190-194.

Bonnet, M., Decety, J., Jeannerod, M., \& Requin, J. (1997). Mental simulation of an action modulates the excitability of spinal reflex pathways in man. Cognitive Brain Research, 5, 221-228.

Byrne, R. W., \& Russon, A. E. (1998). Learning by imitation: A hierarchical approach. Behavioural \& Brain Sciences, 21, 667-684, discussion 684-721.

Decety, J., \& Grèzes, J. (1999). Neural mechanisms subserving the perception of human actions. Trends in Cognitive Sciences, 3, 172-178.

Decety, J., Grezes, J., \& Costes, N. (1997). Brain activity during observation of actions. Influence of action content and subject's strategy. Brain, 120, 1763-1777. 
Di Lazzaro, V., Restuccia, D., \& Oliviero, A. (1998). Effects of voluntary contraction on descending volleys evoked by transcranial stimulation in conscious humans. Journal of Physiology, 508, 625-633.

Fadiga, L., Buccino, G., Craighero, L., Fogassi, L., Gallese, V., \& Pavesi, G. (1999). Corticospinal excitability is specifically modulated by motor imagery: A magnetic stimulation study. Neuropsychologia, 37, 147-158.

Fadiga, L., Fogassi, L., Pavesi, G., \& Rizzolatti, G. (1995). Motor facilitation during action observation: A magnetic stimulation study. Journal of Neurophysiology, 73, 2608-2611.

Gangitano, M., Mottaghy, F. M., \& Pascual-Leone, A. (2001). Phase-specific modulation of cortical motor output during movement observation. NeuroReport, 12, 1489-1492.

Grezes, J., Costes, N., \& Decety, J. (1999). The effects of learning and intention on the neural network involved in the perception of meaningless actions. Brain, 122, 1875-1887.

Grezes, J., \& Decety, J. (2001). Functional anatomy of execution, mental simulation, observation, and verb generation of actions: A meta-analysis. Human Brain Mapping, 12, 1-19.

Hall, C., Buckolz, E., \& Fishburne, G. J. (1992). Imagery and the acquisition of motor skills. Canadian Journal of Sport Sciences, 17, 19-27.

Hari, R., Forss, N., Avikainen, S., Kirveskari, E., Salenius, S., \& Rizzolatti, G. (1998). Activation of human primary motor cortex during action observation: A neuromagnetic study. Proceedings National Academy of Sciences USA, 95, 15061-15065.

Hashimoto, R., \& Rothwell, J. C. (1999). Dynamic changes in corticospinal excitability during motor imagery. Experimental Brain Research, 125, 75-81.

Heyes, C. (2001). Causes and consequences of imitation. Trends in Cognitive Sciences, 5, 253-261.

Iacoboni, M., Koski, L. M., Brass, M., Bekkering, H., Woods, R. P., Dubeau, M. C., Mazziotta, J. C., \& Rizzolatti, G. (2001). Reafferent copies of imitated actions in the right superior temporal cortex. Proceedings National Academy of Sciences USA, 98, 13995-13999.

Iacoboni, M., Woods, R. P., Brass, M., Bekkering, H., Mazziotta, J. C., \& Rizzolatti, G. (1999). Cortical mechanisms of human imitation. Science, 286, 2526-2528.

Jeannerod, M. (2001). Neural simulation of action: A unifying mechanism for motor cognition. Neuroimage, 14, S103-S109.

Jeannerod, M., \& Frak, V. (1999). Mental imaging of motor activity in humans. Current Opinions in Neurobiology, 9, 735-739.

Kasai, T., Kawai, S., Kawanishi, M., \& Yahagi, S. (1997). Evidence for facilitation of motor evoked potentials (MEPs) induced by motor imagery. Brain Research, 744, 147-150.
Maeda, F., Kleiner-Fisman, G., \& Pascual-Leone, A. (2002). Motor facilitation while observing hand actions: Specificity of the effect and role of observer's orientation. Journal of Neurophysiology, 87, 1329-1335.

Mataric, M. J., \& Pomplun, M. (1998). Fixation behavior in observation and imitation of human movement. Cognitive Brain Research, 7, 191-202.

Mellet, E., Petit, L., Mazoyer, B., Denis, M., \& Tzourio, N. (1998) Reopening the mental imagery debate: Lessons from functional anatomy. Neuroimage, 8, 129-139.

Meltzoff, A. N., \& Moore, M. K. (1977). Imitation of facial and manual gestures by human neonates. Science, 198, 74-78.

Mills, K. R., \& Kimiskidis, V. (1996). Cortical and spinal mechanisms of facilitation to brain stimulation. Muscle and Nerve, 19, 953-958.

Mills, K. R., \& Nithi, K. A. (1997). Corticomotor threshold to magnetic stimulation: Normal values and repeatability. Muscle and Nerve, 20, 570-576.

Nielsen, J. F. (1996). Logarithmic distribution of amplitudes of compound muscle action potentials evoked by transcranial magnetic stimulation. Journal of Clinical Neurophysiology, 13, 423-434.

Ortuno, F., Ojeda, N., \& Arbizu, J. (2002). Sustained Attention in a Counting Task: Normal Performance and Functional Neuroanatomy. Neuroimage, 17, 411-420.

Pfurtscheller, G., \& Neuper, C. (1997). Motor imagery activates primary sensorimotor area in humans. Neuroscience Letters, 239, 65-68.

Porro, C. A., Francescato, M. P., \& Cettolo, V. (1996). Primary motor and sensory cortex activation during motor performance and motor imagery: A functional magnetic resonance imaging study. Journal of Neuroscience, 16, 7688-7698.

Rossi, S., Pasqualetti, P., Tecchio, F., Pauri, F., \& Rossini, P. M. (1998) Corticospinal excitability modulation during mental simulation of wrist movements in human subjects. Neuroscience Letters, 243, 147-151.

Rossini, P. M., Rossi, S., Pasqualetti, P., \& Tecchio, F. (1999). Corticospinal excitability modulation to hand muscles during movement imagery. Cerebral Cortex, 9, 161-167.

Strafella, A. P., \& Paus, T. (2000). Modulation of cortical excitability during action observation: A transcranial magnetic stimulation study. NeuroReport, 11, 2289-2292.

Ugawa, Y., Terao, Y., Hanajima, R., Sakai, K., \& Kanazawa, I. (1995). Facilitatory effect of tonic voluntary contraction on responses to motor cortex stimulation. Electroencephalography \& Clinical Neurophysiology, 97, 451-454.

Weber, M., \& Eisen, A. A. (2002). Magnetic stimulation of the central and peripheral nervous systems. Muscle and Nerve, 25, 160-175. 